\title{
UHF Radio Channel Measurements and Prediction for Maritime Communications
}

\author{
F.Salazar-Quiñonez ${ }^{1}$, M. Abdulaziz ${ }^{2}$, A. Hussaini ${ }^{3}$, R.A. Abd-Alhameed ${ }^{4}$, \\ A.Cowley ${ }^{5}$, D. Bladen 6 \\ \{fsalazar@dragonsystemsltd.co.uk ${ }^{1}$, maiwadaabdulaziz@yahoo.com ${ }^{2}$, \\ ash.hussaini@aun.edu.ng ${ }^{3}$,r.a.a.abd@bradford.ac.uk ${ }^{4}$, andy@cowley.eu ${ }^{5}$, \\ derek.bladen@commercial-radio.co.uk $\left.{ }^{6}\right\}$ \\ Dragon Systems LTD, Milford Haven, SA73 2ND, United Kingdom ${ }^{1}$ \\ Mobile and Satellite Faculty of Engineering and Informatics, University of Bradford, Bradford, BD7 \\ 1DP, United Kingdom ${ }^{2,3,4}$, \\ Home Sensor LTD, Milford Haven, SA73 2QN, United Kingdom ${ }^{5}$ \\ Commercial Radio Systems, Cardiff, CF11 8BA, United Kingdom ${ }^{6}$
}

\begin{abstract}
Radio Service Coverage Area of UHF Repeater systems is one of the most important performance elements for a ship to shore radio communications within industries around the world. Each implemented system is designed to fulfill their specific demands, which in this case is balancing coverage limitations and transmission power restrictions due to petrochemical industry regulations. This paper presents the performance of an Analogue UHF Repeater Extender characterized by a 3D Shoot and Bounce Ray Tracing Propagation Model combining efforts of field strength distribution, time of arrival, and propagation-channel environment. The work is focused on the extender's field measurements results in comparison to a Wireless InSite 3D SBR simulation model.
\end{abstract}

Keywords: UHF Repeater Extender, Marine Communications, Ship to Shore, 3D SBR, Shoot, and Bounce Ray Tracing Technique, ATEX Radios, LNG Cargo.

\section{Introduction}

Reliable Maritime Radio Communications for any Military, Industrial or Private Cargo Vessel and the port ashore it's crucial for safe coordination of manoeuvres and operations such as docking, sailing, loading, unloading, as well as handling emergencies. Depending on each situation the communication quality amongst the crew members, coordinators, and the control room is paramount to perform risk-free tasks [1]. Each cargo vessel holds goods and/or materials that could be in nature hazardous such as petrochemicals or could represent a hazard if not handled correctly, for example, military equipment, heavy cargo containers. In this case, significant degradation of the signal is experienced when radio communications try to penetrate from the shore control room using UHF radio repeaters to handheld UHF radios inside the control room from the ship.

In a petrochemical cargo tanker, this degradation of the signal is present due to a mixture of factors such as the complexity of the metallic structure of the vessel as well as the transmit 
power limitation due to ATEX regulations for communications within hazardous explosive atmospheres that applies to the vessel as well shore equipment [2].

\subsection{Studies on Measurements}

Several studies have been developed over many years to evaluate the radio wave propagation from maritime vessels communicating with the shore for different applications. [3-6]. Some literature has experimented on theoretical analysis against measurement campaigns for a line of sight (LOS) and non-line of sight (NLOS) Scenarios [4-6] implementing antenna diversity or MIMO antennas network to extend the coverage of the communications within the ship [7] as well as ad-hoc network system for multiple vessels [8] for security and positioning purposes [9$10]$.

\subsection{Studies on Simulation}

The use of radio propagation simulation software on large scenarios facilitates a relatively fast estimation of the channel characteristics, such as the power delay profile, signal field distribution, time of arrival, among others [11-18]. The following studies have simulated radio repeater propagation environments [11], using 3D Simulation software to evaluate the radio frequency propagation on outdoor to indoor scenarios for high-speed communications [12-13] as well as a vessel to harbour communications using a Shoot and bounce ray-tracing technique [14] with different vessel location LOS and NLOS. Using techniques have a better resolution importing computer-aided design CAD models for the complex structure of the ship [15].

\subsection{Studies on Simulation vs Measurements}

A combination of Measurements Campaigns and Simulation models have been applied to some studies to corroborate results in oversea radio propagation [16-18] using radio channels in frequencies above the $5 \mathrm{GHz}$ [17-18] to investigate the Line-of-Sight propagation and Non-Line of Sight standoff distances for different size of vessels measuring power delay profile (PDP) and comparing it with 3D propagation models. In the same way, the paper [18] analyses channel characteristics of the UHF band $(225-450 \mathrm{MHz})$ onboard a merchant ship providing a good representation for the shape of the impulse response for a cluttered environment. Investigations such as [19] demonstrate that the Wireless InSite 3D simulation tool provides a good estimation of the measurement results.

\section{Repeater Extender System}

The idea presented in this paper to overcome the lack of signal without affecting the system's transmission power will be achieved by implementing an innovative design of an extender repeater radio system array. This system will be located at the top of the jetty or berth building, closer to the vessel, and will interact with the existing repeater system. The new system will relay the signal from within the main system's range creating a new and stronger radio transmission able to find a path to reach inside the ship as well as to be close enough to receive the transmissions from the vessel's handhelds. 


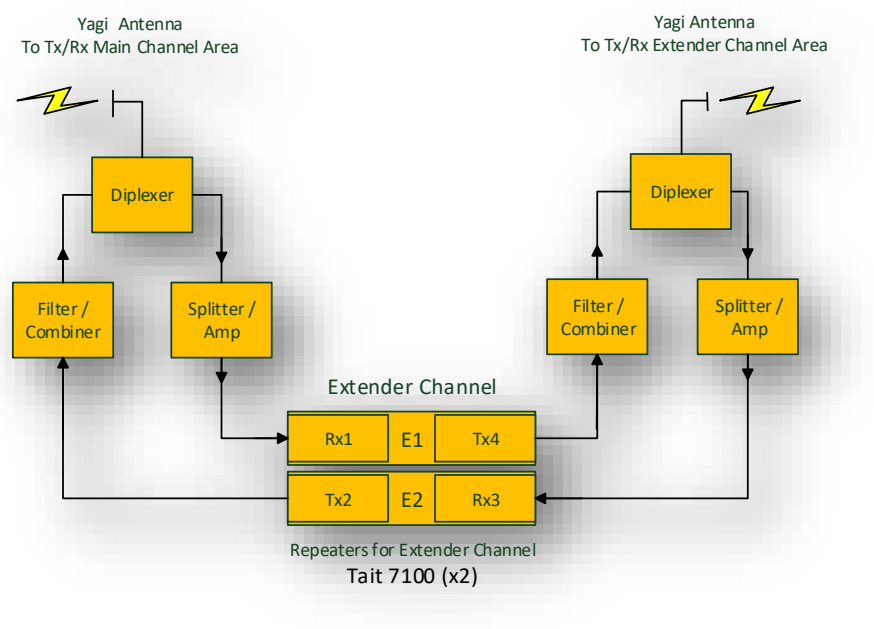

Fig. 1. Extender Array.

The extender array consists of two radio repeaters TAIT 7100 (for this case study), Rx1/Tx2 working together to emulate a normal portable radio, and $\mathrm{Rx} 3 / \mathrm{T} \times 4$ working as a normal repeater. Radio Repeater "E1" receives the transmission signal on frequency 1 (Rx1) from the Main Channel and re-transmits on Tx4 into the Extender Channel to communicate to handheld radios on this channel. "E2" receives the signal Rx3 from the handheld radios on the Extender Channel and retransmits to Tx2 to the main repeater as represented in Fig 1. Once the re-transmission from the Extender Repeater reaches the Main repeater, it is then broadcasted on Tx1 to the handheld radios on the Main Channel as well as a return of transmission to "E1". This system allows handheld radios to intercommunicate on two different channels at different coverage areas as shown in Fig 2. Four Element Yagi antennas were located at the top of a jetty port control building (10m height) on a $3 \mathrm{~m}$ pole and connected to the diplexer using cable RG 213/U. A bird thru line wattmeter tester was utilised to verify that $2 \mathrm{~W}$ was the power transmitted at the antenna level, as per ATEX regulations [2]. In the same way, the handheld radios' maximum transmission power is $1 \mathrm{~W}$ by design.

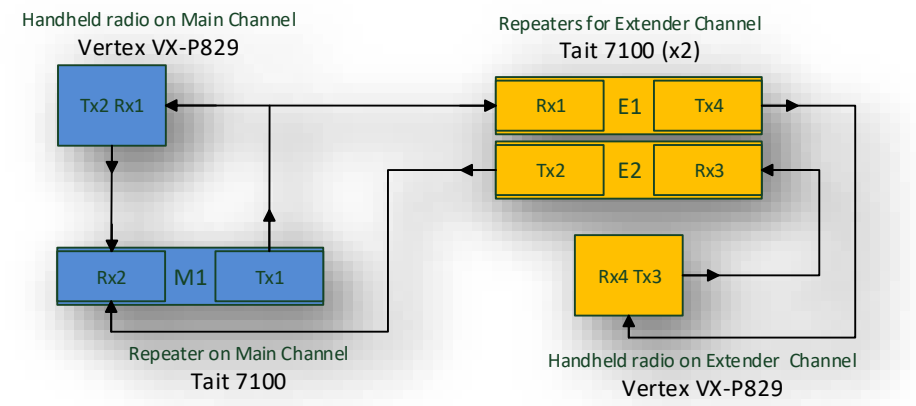

Fig. 2. Extender Transmission System. 


\section{Measurement Setup}

The measurements were accomplished using a Field Strength Analyser Protek $3290 \mathrm{~N}$ to simulate a handheld radio, this has been calibrated to analyse narrowband signals at $12.5 \mathrm{kHz}$ Channel Bandwidth and specifically for frequencies 1 and $4(\mathrm{Rx} 1, \mathrm{Rx} 4)$. The sensitivity range was set from $0-120 \mathrm{~dB}$ and localised at $1.5 \mathrm{~m}$ from the floor on each test location. Due to weather factors that may affect the received signal, this campaign was done on a clear and dry day. Date and time were also recorded to evaluate the tide level and relative position to locate the height of the ship about the shore side, for modelling references [20].

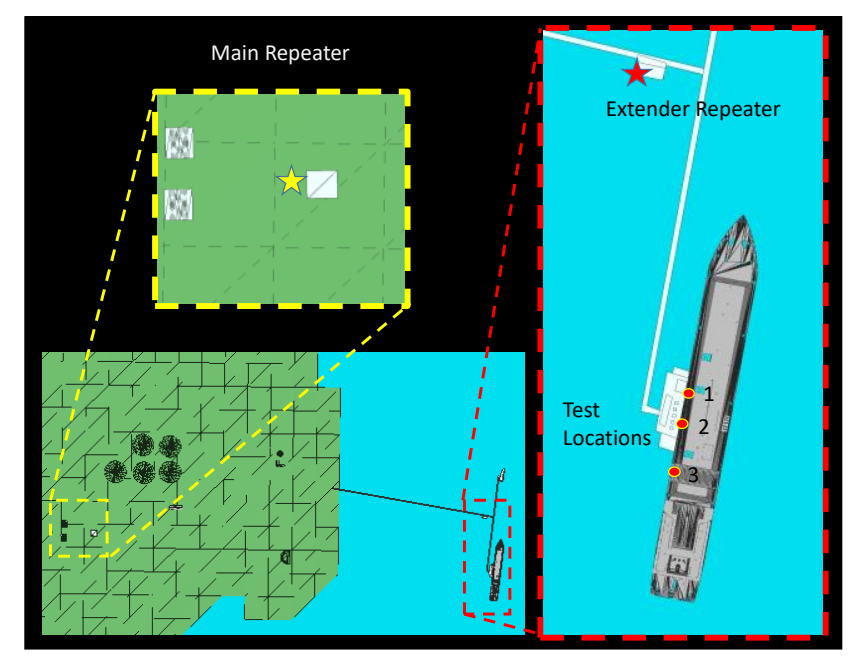

Fig. 3. Scenario Layout

In Fig 3, the yellow star represents the Main Repeater Antenna, and the red star the Extender Repeater antenna. The Field Strength Analyser was tested along with three different locations on the ship. Test locations " 1 " and " 2 " are located outside the cargo, where unload operations require reliable communications, and test location " 3 " is located inside the control room from the ship. The received signal strength index (RSSI) was measured at these three locations on transmissions from the Main repeater system as well as the Extender Repeater system.

\section{Simulation Model}

A simulation 3D model was created to estimate and co-validate the performance of the proposed Extender system. The model was created using Wireless InSite software working on a Study Area of $2157.43 \mathrm{~m}$ x $4339.97 \mathrm{~m}$ x 146m. The terrain profile from the land was uploaded from Google Earth using Google Sketchup 7.0 with the application of the Geo-Location tool as shown in Fig 4 [21]. 


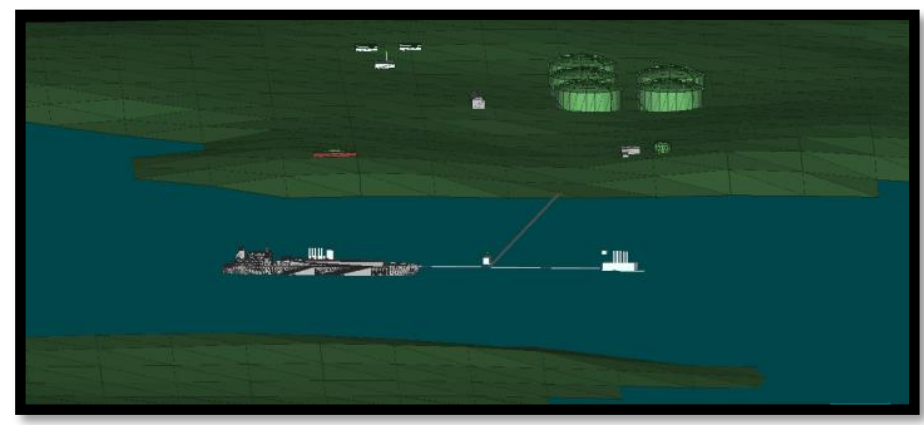

Fig. 4. Terrain profile.

The rest of the buildings, the jetty trestle, the fort (in red), the tank farm, and the ship were also created on Sketchup 7.0, converted into objects, and imported into the Wireless InSite model as shown in Fig 5. The size of each of the objects was also corroborated with their correspondent structural drawings to match each of the features and sizes to the real-world scenario. The ship and the Tank farm designs were designed available from a 3D warehouse and modified to fit the study purposes [22].

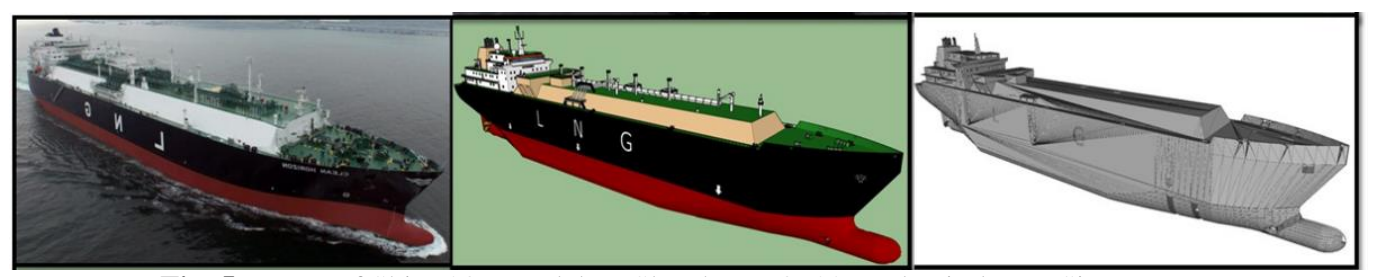

Fig. 5. Image of Ship [23], Model on Sketchup 7.0 [22] and Wireless InSite.

\subsection{Materials}

As the integrity of all the elements is crucial in the investigation, therefore the material specifications were taken from the Remcom database library [24]. Terrain profile is set up as wet earth, buildings as concrete, and the fort of brick material. The Ship and the tank farm will be considered as perfect electric conductors (PEC).

Table 1. Materials [24].

\begin{tabular}{|l|l|l|l|}
\hline Material & Permittivity & Conductivity $(\mathrm{s} / \mathrm{m})$ & Thickness $(\mathrm{m})$ \\
\hline Concrete & 15 & 0.015 & 0.3 \\
\hline Wet Earth & 25 & 0.02 & 0 \\
\hline Brick & 4.44 & 0.001 & 0.125 \\
\hline Sea Water & 81 & 20 & 0 \\
\hline
\end{tabular}

\subsection{Ship}

To have an accurate location of the vessel in the model, there are a couple of important factors to consider. One is the tide level and the second is the relative position of the ship against the berth. The tide level at the time of measurements was $4.48 \mathrm{mts}$ to locate the ocean level 
regarding the height of the Jetty and the berth. In the same way, the relative position of the ship regarding the shore was $-5.1 \mathrm{mts}$. This was done measuring from the vessel's upper deck level to the upper platform from the shore side. This is important to consider because a ship unloading material loses weight from the cargo, therefore it increases its floating capabilities meaning that rises gradually from the water.

\subsection{Antennas and Locations}

The transmit antennas in the main channel consist of a Half-wave dipole antenna transmitting at $33 \mathrm{dBm}$ to represent a four-element stacked array dipole antenna mounted on a $22 \mathrm{~m}$ height mast. In extender system will transmit at $33 \mathrm{dBm}$ using a Directional dipole antenna to simulate a 4 element Yagi antenna mounted on a $3 \mathrm{~m}$ pole on top of the Jetty Building.

The receive antennas that will simulate handheld radios will be represented by short dipole antennas at different test locations. These antennas will have the same sensitivity range as the real test measurements of $0-120 \mathrm{dBm}$. Test location scenarios for the receivers 1,2 (both outside) and 3 (inside) on the measurement campaign will be represented by Rx Out 1, Rx Out 2, and Rx IN in the simulation, respectively as shown in Fig 6.

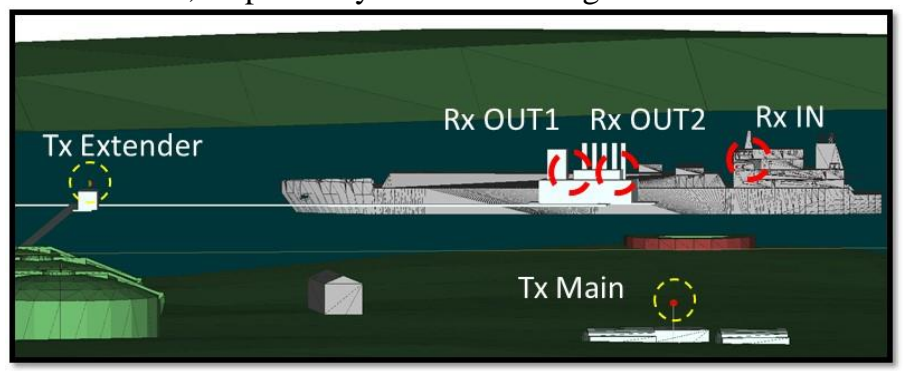

Fig.6. Antenna Positioning.

Additional losses were introduced into the study due to cable type, length, connectors, and couplers for the Main and the Extender antennas connection.

Table 2. Antenna Losses.

\begin{tabular}{|l|l|l|l|}
\hline \multicolumn{3}{|l|}{ Main Antenna } & Extender Antenna \\
\hline LDF - 550 & Loss & RG213/U & Loss \\
\hline $50 \mathrm{mts}$ & $1.5 \mathrm{~dB}$ & $30 \mathrm{mts}$ & $5 \mathrm{~dB}$ \\
\hline Connector $(\mathrm{x} 2)$ & $2 \mathrm{~dB}$ & Connector $(\mathrm{x} 2)$ & $3 \mathrm{~dB}$ \\
\hline Coupler & $1.5 \mathrm{~dB}$ & Coupler & $2 \mathrm{~dB}$ \\
\hline
\end{tabular}

From Table 2 it was deduced that the Main antenna cable loss calculation is approximately 5 $\mathrm{dB}$, and the Extender antenna cable loss is calculated to be at least $10 \mathrm{db}$.

\subsection{Study Area}

The model calculations on Wireless InSite were performed using the Full 3D model. This model is based upon the shoot and bounces ray-tracing technique in a $3 \mathrm{D}$ model using $0.25^{\circ}$ ray spacing, 6 reflections, 4 transmissions, and 1 diffraction. This allowed the calculation of the path loss through the interaction of the transmitted rays with the basic multipath mechanisms 
(transmission, reflection, diffraction, and scattering) and evaluating the rays that reached the receiver.[25]

\section{Results}

The measurement campaign was performed using a Signal Strength Analyser to simulate a handheld radio and capture the Received Signal Strength Indicator (RSSI) from the Main Transmitter as well as the Extender Transmitter. These measurements were evaluated at 3 locations, two outside the ship and one inside the ship, each at $1.5 \mathrm{~m}$ from the floor. The readings at each location were averaged for simpler analysis. The 3D simulation model was created using Wireless InSite and performed accurate calculations using the shoot and bounce ray-tracing technique. The 3D SBR ray-tracing technique applies an image method approach to capture a large scenario precisely. The calculation time of the simulation time was done in $4 \mathrm{hrs}$ and 35 mins using PC intel core duo @ 4GB of RAM. Transmissions from Main and Extender created multipath that reached the receivers located outside and inside the ship. There was not any Lineof-Sight transmission able to reach the receiver inside the ship as represented in Figs 7 and 8.

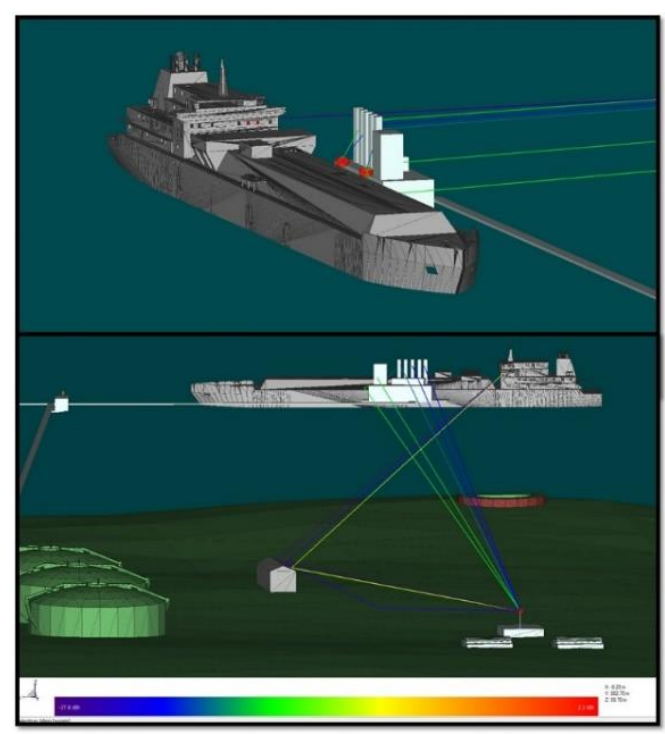

Fig. 7. Main Tx to Ship Rx.

Fig 7 shows the few transmission paths from the Main Transmitter that reached the receivers on the Ship. Structural buildings interact as obstructions or multipath features for the ray trajectory. The signal received on the ship from this calculation fluctuates from $-79 \mathrm{dBm}$ to $-86 \mathrm{dBm}$ (averaged values) which represents the low numbers of rays received, therefore the lack of strong or reliable communications. 


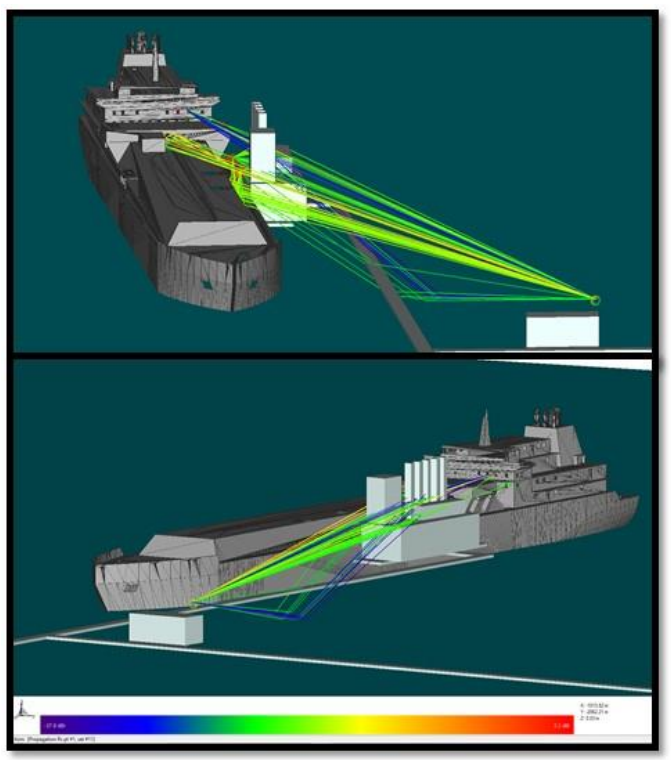

Fig. 8. Extender Tx to Ship Rx.

With the implementation of the Extender system closer to the ship, the results showed an increased number of rays reaching the Ship's receivers as shown in Fig 8 despite the location of the control tower, loading arms, high-pressure pumps, and pipework that could be obstructing the signal's path. As well as the higher quantity of paths reaching the receivers a stronger signal is received inside the ship, improving with this the quality and reliability of communications. The range of the signal received is from $-58 \mathrm{dBm}$ to $-66 \mathrm{dBm}$ (mean values).

Table 3. Mean Measurements vs Mean Simulation Results.

\begin{tabular}{|l|l|l|l|l|l|l|l|}
\hline \multirow{2}{*}{ Tx/Rx } & Rx OUT 1 & Rx OUT 2 & \multicolumn{1}{l|}{ Rx IN } \\
\cline { 2 - 7 } & Simulation & RSSI & Simulation & RSSI & Simulation & RSSI \\
\hline Tx Main (dB) & -79.2763 & -80 & -84.9540 & -86 & -86.3110 & -85 \\
\hline Tx Extender (dB) & -58.9581 & -58 & -59.3064 & -61 & -66.2826 & -64 \\
\hline \multicolumn{7}{|l|}{} \\
\hline Improvement (dB) & 20.3182 & 22 & 25.6476 & 25 & 20.0284 & 21 \\
\hline
\end{tabular}

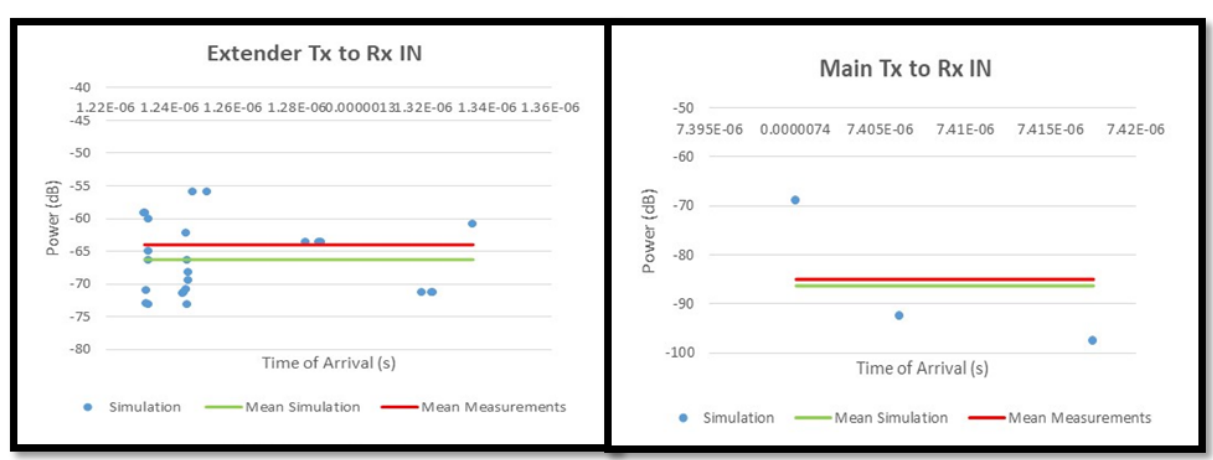

Fig. 9. Power vs ToA to Rx IN. 
Table 3 shows the compilation of the RSSI from the measurements and the simulation at each of the locations. This table compares the simulation against the measurement and shows an improvement of a minimum of $20 \mathrm{~dB}$ from the Extender system against the Main System.

Fig 9 demonstrate visually the proximity of the mean RSSI values from the measurements and the mean signal strength received inside the ship on the simulation including the intensity of rays at each system.

\section{Conclusion}

The implementation of the UHF Extender Repeater System had a strong impact on the communications with the ship, making more reliable transmissions and increasing the coverage area. Once compared with the RSSI from the UHF Main Repeater system the measurements of the field strength distribution showed that the Extender system provided a minimum of $20 \mathrm{~dB}$ improvement on RSSI inside the control room in the ship. The implementation of the 3D SBR simulation model using Wireless InSite software provided a good estimation of the channel propagation without exhausting computational effort. The comparison between the results obtained from the simulation data and the measurements had a high correlation of $99.25 \%$. Despite the high accuracy of the model, the complexity of the plant design limits the possibility of it being used in the future. Further improvements could be made to the extender system in the future to reduce losses from cable and connectors.

\section{References}

[1] H. Karahalios, "The Severity of Shipboard Communication Failures in Maritime Emergencies: A Risk Management Approach," International Journal of Disaster Risk Reduction, 2018.

[2] T. Jespen "ATEX - Ignition Sources. In: ATEX - Explosive Atmospheres," Springer Series in Reliability Engineering. Springer, Cham. 2016

[3] Y. M. Le Roux, J. Ménard, C. Toquin, J. P. Jolivet and F. Nicolas, "Experimental measurements of propagation characteristics for maritime radio links," 2009 9th International Conference on Intelligent Transport Systems Telecommunications, (ITST), Lille, 2009, pp. 364-369.

[4] J. Yu, W. Chen, K. Yang, C. Li, F. Li, and Y. Shui, "Path Loss Channel Model for Inland River Radio Propagation at 1.4 GHz," International Journal of Antennas and Propagation, vol. 2017, Article ID 5853724, 15 pages, 2017.

[5] K. Yang, T. Roste, F. Bekkadal, K. Husby and O. Trandem, "Long-Distance Propagation Measurements of Mobile Radio Channel over Sea at $2 \mathrm{GHz}$," 2011 IEEE Vehicular Technology Conference (VTC Fall), San Francisco, CA, 2011, pp. 1-5.

[6] K. Yang, T. Roste, F. Bekkadal and T. Ekman, Land-to-Ship Radio Channel Measurements over Sea at $2 \mathrm{GHz}$," 2010 6th International Conference on Wireless Communications Networking and Mobile Computing (WiCOM), Chengdu, 2010, pp. 1-4.

[7] V. J. Dwivedi, \& N. A. Kotak, "Simulation and Performance Analysis of Blast Structure in MIMO Environment," International Journal of Research in Engineering and Technology. April 2014.

[8] X. Su, B. Hui, K. Chang \& S. Kim. "Improvement of the Link Reliability for Ship Ad-Hoc Network by Employing Multiple Antennas," The Journal of Korea Information and Communications Society, 2012.

[9] H. Luo, K. Wu, Z. Guo, L. Gu and L. M. Ni, "Ship Detection with Wireless Sensor Networks," in IEEE Transactions on Parallel and Distributed Systems, vol. 23, no. 7, pp. 1336-1343, July 2012.

[10] B. Hui, K. Jeon, K. Chang, S. Kim, J. Park and Y. Lim, "Design of radio transmission technologies for VHF band ship ad-hoc network," ICTC 2011, Seoul, 2011, pp. 626-629. 
[11] A. Hastings, "Modeling of RF propagation in a radio repeater environment," 2016 IEEE/ACES International Conference on Wireless Information Technology and Systems (ICWITS) and Applied Computational Electromagnetics (ACES), Honolulu, HI, 2016, pp. 1-2.

[12] E. Semaan, F. Harrysson, A. Furuskär and H. Asplund, "Outdoor-to-indoor coverage in high frequency bands," 2014 IEEE Globecom Workshops (GC Wkshps), Austin, TX, 2014, pp. 393-398.

[13] W. Manan, H. Obeidat, A. Al-Abdullah, R. Abd-Alhameed and F. Hu "Indoor to Indoor And Indoor To Outdoor Millimeter Wave Propagation Channel Simulations At 26 Ghz, 28 Ghz And 60 Ghz For 5 G Mobile Networks" The International Journal of Engineering and Science (IJES), Volume 7, Issue 3, Ver. II, Pages PP 08-18, 2018

[14] J.K. Infantolino, K. L. Labowski, "Complex 3D Modeling of Sea to Land Scenario" 29th Annual Review of Progress in Applied Computational Electromagnetics, ACES, 2013/3/24.

[15] L.E. Rodriguez-Gallo "Propagation Modeling od Wireless Systems on Shipboard External decks," MSc Thesis Naval Postgraduate School, Monterey, California. USA. 2006.

[16] Y. H. Lee, F. Dong, Y. S. Meng. "Stand-off distances for non-line-of-sight maritime mobile applications in 5GHZ band". Progress In Electromagnetics Research, 2013, pp 321-336.

[17] F. Dong and Y. H. Lee, "Non-line-of-sight communication links over sea surface at 5.5GHz," AsiaPacific Microwave Conference 2011, Melbourne, VIC, 2011, pp. 1682-1685.

[18] X. H. Mao and Y. H. Lee, "UHF Propagation Along a Cargo Hold on Board a Merchant Ship," in IEEE Transactions on Wireless Communications, vol. 12, no. 1, pp. 22-30, January 2013.

[19] Y. Dama, R. Abd-Alhameed, F. Salazar-Quiñonez, D. Zhou, S. Jones and S. Gao, "MIMO indoor propagation prediction using 3D shoot-and-bounce ray (SBR) tracing technique for $2.4 \mathrm{GHz}$ and 5 $\mathrm{GHz}$," Proceedings of the 5th European Conference on Antennas and Propagation (EUCAP), Rome, 2011, pp. 1655-1658.

[20] S. Balkees P A, K. Sasidhar and S. Rao, "A survey-based analysis of propagation models over the sea," 2015 International Conference on Advances in Computing, Communications and Informatics (ICACCI), Kochi, 2015, pp. 69-75.

[21] T. S. Rappaport, J. N. Murdock, D. G. Michelson and R. Shapiro, "An Open-Source Archiving System," in IEEE Vehicular Technology Magazine, vol. 6, no. 2, pp. 24-32, June 2011.

[22] https://3dwarehouse.sketchup.com/model/u3c83a3e6-67504410-80a7-af656dc0298b/LNG-carrier, Fabio D.

[23] www.dynagas.com

[24] Remcom, The Wireless Insite User's Manual Release 3.1.0.1. Pennsylvania, USA: Remcom Inc. , 2017.

[25] P. Pajusco, "Propagation channel models for mobile communication," Comptes Rendus Physique, vol. 7, pp. 703-714, 2006. 